\title{
Morphology-induced oscillations of the electron-spin precession in Fe films on $\mathrm{Ag}(001)$
}

\author{
L. Tati Bismaths, ${ }^{1}$ L. Joly, ${ }^{2}$ A. Bourzami,${ }^{1}$ F. Scheurer,${ }^{1}$ and W. Weber ${ }^{1}$ \\ ${ }^{1}$ Institut de Physique et Chimie des Matériaux de Strasbourg, UMR 7504, ULP CNRS, 23 rue du Loess, Bô̂te Postale 43, \\ F-67034 Strasbourg Cedex 2, France \\ ${ }^{2}$ Paul Scherrer Institut, Swiss Light Source, WSLA/213, CH-5232 Villigen PSI, Switzerland \\ (Received 27 March 2008; revised manuscript received 29 May 2008; published 18 June 2008)
}

\begin{abstract}
Spin-polarized electron reflection experiments on Fe films on $\mathrm{Ag}(001)$ show oscillations of the electron-spin precession as a function of the Fe thickness with monolayer periodicity. They are attributed to morphological changes of the Fe film. This shows-because of total angular momentum conservation-that the transfer of spin-angular momentum from the incident electrons to the ferromagnetic film can be extremely sensitive to the morphology and structure of the film.
\end{abstract}

DOI: 10.1103/PhysRevB.77.220405

PACS number(s): 75.70.Cn, 75.70.Rf, 78.67.De, 72.25.Mk

The configuration of magnetization orientation in a metallic ferromagnetic system can strongly affect the electron transport properties of the system, a phenomenon known as the "giant magnetoresistance" effect. 1,2 Transfer of spin angular momentum, on the other hand, represents the reverse effect: the influence of a spin-polarized current on the magnetization of the ferromagnetic system. The phenomenon originates from the exchange of angular momentum between a polarized current and the magnetization, a concept that has been put forward by Slonczewski ${ }^{3}$ and Berger. ${ }^{4}$ In fact, this relatively new phenomenon has been proven to be a way of reversing the magnetization without the application of a magnetic field. ${ }^{5}$ Although much attention has been devoted to this topic, all efforts focused on experiments involving the spin transfer from electrons from one solid medium to another. While much insight has been obtained in these kinds of experiments, the spin-transfer process could be made clearer if the spin states of the incoming and outgoing electrons, which are usually not well known in these experiments, could be assessed by some known independent means. This is possible in experiments in which the spin polarization of the electrons is measured before and after the interaction with a ferromagnetic film. Because of total angular momentum conservation, such experiments allow us to evidence the spin-transfer effect by measuring the spin motion of the electrons that have been interacting with the ferromagnetic film. In the past, this technique has been used by us both in transmission and in reflection geometry in order to identify the parameters influencing the spin-transfer effect. Although the current densities used in this technique are too small by many orders of magnitude to induce a sizeable effect on the magnetization vector, the torque per electron exercised on the magnetization can be determined. The measurements in transmission showed that the precessional motion of the incident electron spin and consequently that of the magnetization is directly determined by the exchange energy. ${ }^{6}$ The experiments in reflection, on the other hand, showed that angular momenta comparable to those in transmission geometry are transferred to the ferromagnet. ${ }^{7}$ In particular, the importance of gaps in the electronic band structure of the ferromagnet ${ }^{7}$ and of quantum-interference effects $^{8,9}$ has been underlined. Here we show that the spin transfer can be influenced strongly via a change of the spindependent electron reflection amplitudes by the morphology and structure of the ferromagnetic film-an effect that has not yet been addressed either theoretically or experimentally. This effect is evidenced upon spin-polarized electron reflection on Fe films on $\operatorname{Ag}(001)$.

The experiment consists of a polarized electron source, a ferromagnetic film that is magnetized remanently in-plane by a magnetic field pulse, and a spin detector (see inset in the bottom panel of Fig. 1). A $70 \%$ polarized electron beam is obtained from an optically pumped $\mathrm{Ga}_{x} \mathrm{P}_{1-x} \mathrm{As}$ superlattice structure with circularly polarized light of $780 \mathrm{~nm}$ wavelength. The electron beam is incident at $45^{\circ}$ with respect to the sample surface with the in-plane projection of the wave vector along the [100] direction of the Fe film. To observe a maximum spin motion, the spin-polarization vector $\mathbf{P}_{0}$ of the incident electrons must be oriented perpendicularly with respect to the magnetization $\mathbf{M}$ of the ferromagnetic film. ${ }^{6}$ It is only in a noncollinear geometry that the magnetization can exert a torque on the spin-polarization vector. Upon reflection on the sample, the specular beam passes through a retarding field energy analyzer. The spin polarization of the elastically scattered electrons, to which we restrict our discussion, is measured by a spin detector.

The origin of the electron-spin motion in ferromagnetic films is the spin-dependent scattering of electrons within the film and at its surface. Supposing a completely polarized electron beam with $\mathbf{P}_{0}$ perpendicular to $\mathbf{M}$, the spin part of the incident electron wave function is described by a superposition of a majority-spin and a minority-spin wave function with equal amplitudes: $\chi_{0} \propto(1,0)+(0,1)$. Because of spin-dependent scattering, the spin wave function of the electron beam after reflection from the ferromagnetic film reads $\chi^{\propto}\left(\left|r^{\uparrow}\right| \exp \left(i \theta^{\uparrow}\right),\left|r^{\downarrow}\right| \exp \left(i \theta^{\downarrow}\right)\right)$, where $\left|r^{\uparrow, \downarrow}\right|$ and $\theta^{\uparrow, \downarrow}$ are, respectively, the moduli and the phases of the spin-dependent reflection amplitudes. This change of the spin wave function corresponds to a precession of $\mathbf{P}$ around $\mathbf{M}$ by an angle $\varepsilon$ $=\theta^{\downarrow}-\theta^{\uparrow} \quad$ and a rotation by an angle $\phi$ $=\arctan \left[\left(\left|r^{\uparrow}\right|^{2}-\left|r^{\downarrow}\right|^{2}\right) / 2\left|r^{\uparrow}\right|\left|r^{\downarrow}\right|\right]$ in the plane spanned by $\mathbf{P}$ and $\mathbf{M}$ with $\left|r^{\uparrow, \downarrow}\right|^{2}=I^{\uparrow, \downarrow}$ the spin-dependent reflectivity (see inset in the bottom panel of Fig. 2). In the following, the values of $\phi$ are always normalized to a fully polarized electron beam.

Prior to Fe deposition, the $\mathrm{Ag}(001)$ crystal is cleaned by several cycles of Ar-ion sputtering and annealing at $800 \mathrm{~K}$ until sharp low-energy electron diffraction (LEED) reflexes 
are visible and no contamination of the surface is detected by Auger spectroscopy. $\mathrm{Fe}$ is deposited at room temperature from an Fe rod heated by electron beam bombardment. During deposition, the pressure is below $3 \times 10^{-10}$ mbar. Nevertheless, we always detect a slight oxygen contamination of the Fe films (0.6 at. \%).

In our electron scattering experiments, the Fe films are grown as follows. At a growth rate of $0.1 \mathrm{~nm} / \mathrm{min}$ we deposit Fe during $30 \mathrm{~s}$. This is followed by a measurement that takes several minutes. Then, the procedure is repeated. Consequently, the average deposition rate is much smaller than $0.1 \mathrm{~nm} / \mathrm{min}$. Auger experiments performed in the same manner show a decrease of the $\operatorname{Ag}(351 \mathrm{eV})$ Auger-line intensity that is well fitted by an exponential decay. As the fitted attenuation length of $0.6 \mathrm{~nm}$ agrees well with the expected one, ${ }^{10}$ the decrease of the Ag-Auger intensity is consistent with a layer-by-layer growth of $\mathrm{Fe}$ on $\mathrm{Ag}(001)$ under our particular growth conditions.

However, islands are formed when the Fe film is deposited at a higher average deposition rate, for instance by increasing the time during which Fe is deposited without interruption. The Auger experiments show a clear tendency: the higher the average deposition rate, the higher the Ag signal at a given Fe thickness. This may explain certain controversies in the literature about the growth of Fe on $\mathrm{Ag}(001) .{ }^{11-14}$

Figure 1 shows the electron reflectivity as a function of $\mathrm{Fe}$ thickness for three different primary electron energies. At $6.5 \mathrm{eV}$, oscillations with a period of one monolayer (1 ML $=0.143 \mathrm{~nm}$ ) are seen. While their amplitude is strong for low coverages, it decreases rapidly with increasing thickness but stays then almost constant (see the inset in the top panel of Fig. 1). At $7 \mathrm{eV}$, the ML oscillations are only visible at low coverages up to $1 \mathrm{~nm}$. For energies above $9 \mathrm{eV}$, no ML oscillations can be identified. Instead we observe one large peak around $0.5 \mathrm{~nm}$. The same structure appears already at lower electron energies but superimposed on the ML oscillations. This structure is attributed to the creation of a standing electron wave between the $\mathrm{Fe}$ surface and the $\mathrm{Fe} / \mathrm{Ag}$ interface.

Figures 2 and 3 show, respectively, the precession angle $\varepsilon$ and the rotation angle $\phi$ as a function of the Fe thickness for three different primary electron energies. The onset of the magnetic signal is independent of the electron energy at about 4 ML. In fact, Fe films on $\mathrm{Ag}(001)$ exhibit a reorientation transition of the magnetization from out-of-plane to in-plane around this thickness. ${ }^{15}$ As our experimental setup does not allow us to magnetize the sample out-of-plane, a magnetic signal appears only when the magnetization is inplane. For thicknesses above $4 \mathrm{ML}$, oscillations of both $\varepsilon$ and $\phi$ are seen. A Fourier analysis of the data for energies between 6 and $9 \mathrm{eV}$ reveals the existence of two periods, a short one with a wavelength of one ML independent of the energy and a longer one whose wavelength varies as a function of the energy (see inset in the bottom panel of Fig. 3). For energies above $9 \mathrm{eV}$, the ML oscillations disappear, while oscillations with the long period remain unchanged.

Beside the occurrence of ML oscillations, we make another interesting observation. While for energies above $9 \mathrm{eV}$ both $\varepsilon$ and $\phi$ saturate for thicknesses of the order of the penetration depth of the electrons $(1-2 \mathrm{~nm})$, this is not the

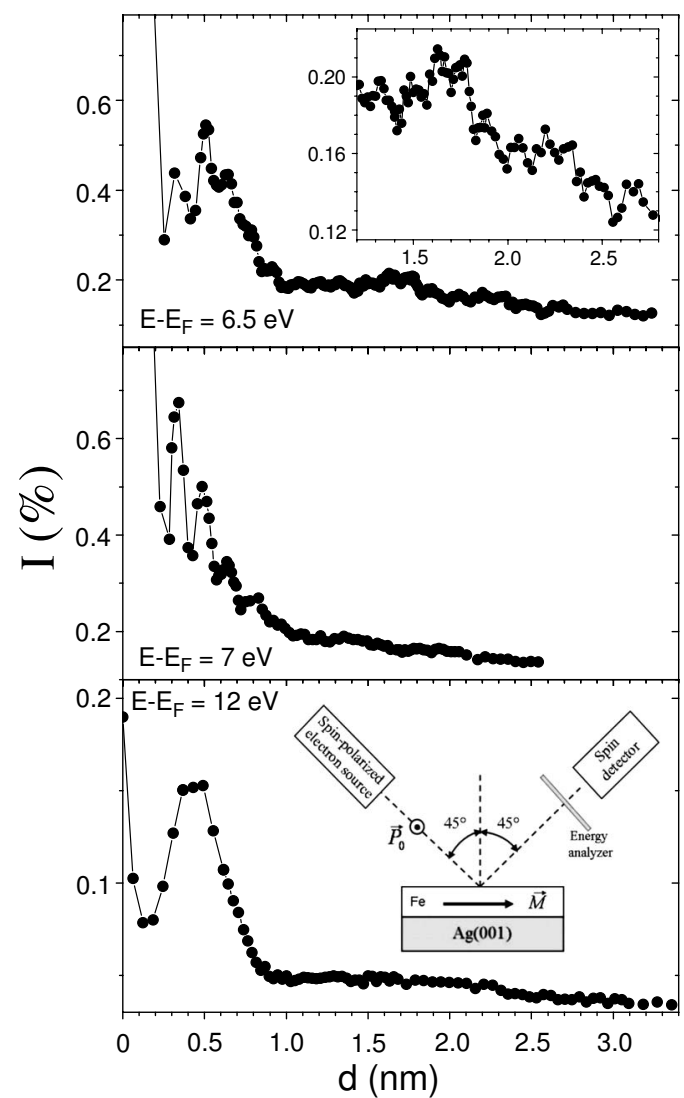

FIG. 1. The spin-integrated electron reflectivity $I$ as a function of the Fe film thickness for three different primary electron energies $E-E_{F}$. The inset in the top panel is a zoom. The lines are guides to the eye. The inset in the bottom panel shows a scheme of the experiment, which consists of a polarized electron source, an in-plane remanently magnetized Fe film, a retarding field energy analyzer, and a spin detector. The incident polarization vector $\mathbf{P}_{0}$ is perpendicularly oriented with respect to the film magnetization $\mathbf{M}$.

case for energies between 6 and $9 \mathrm{eV}$. Instead, we find an overall change in both $\varepsilon$ and $\phi$ as a function of the Fe thickness that is linear over a large thickness range. Then the slope becomes smaller and the signal levels off for thicknesses above $9 \mathrm{~nm}$ (see inset in the middle panel of Fig. 2). It is interesting to note that the existence of an overall change with thickness is accompanied by ML oscillations (see 6.5 and $7 \mathrm{eV}$ ) that disappear when the overall slope is vanishing (see $12 \mathrm{eV}$ ). We will show in the following that these two phenomena are related.

Before discussing the possible origins of the ML oscillations, let us first discuss the occurrence of the long-period oscillations. In order to understand them, one has to consider a multiple-reflection model that is analogous to the FabryPérot interferometer model used in optics. In our recent work on nonmagnetic films $(\mathrm{Cu}, \mathrm{Au})$ deposited on a ferromagnetic $\mathrm{Co}(001)$ substrate, 8,9 we demonstrated that the quantuminterference effects that appear in all three measured quantities, i.e., the reflected intensity, $\varepsilon$, and $\phi$, can be well described within the Fabry-Pérot interferometer model. Whereas in $\mathrm{Cu}$ or $\mathrm{Au}$ films on $\mathrm{Co}$ the electrons traveling in the nonmagnetic layer have a spin-independent wave vector, the latter is spin-dependent in Fe films on Ag. We thus expect 


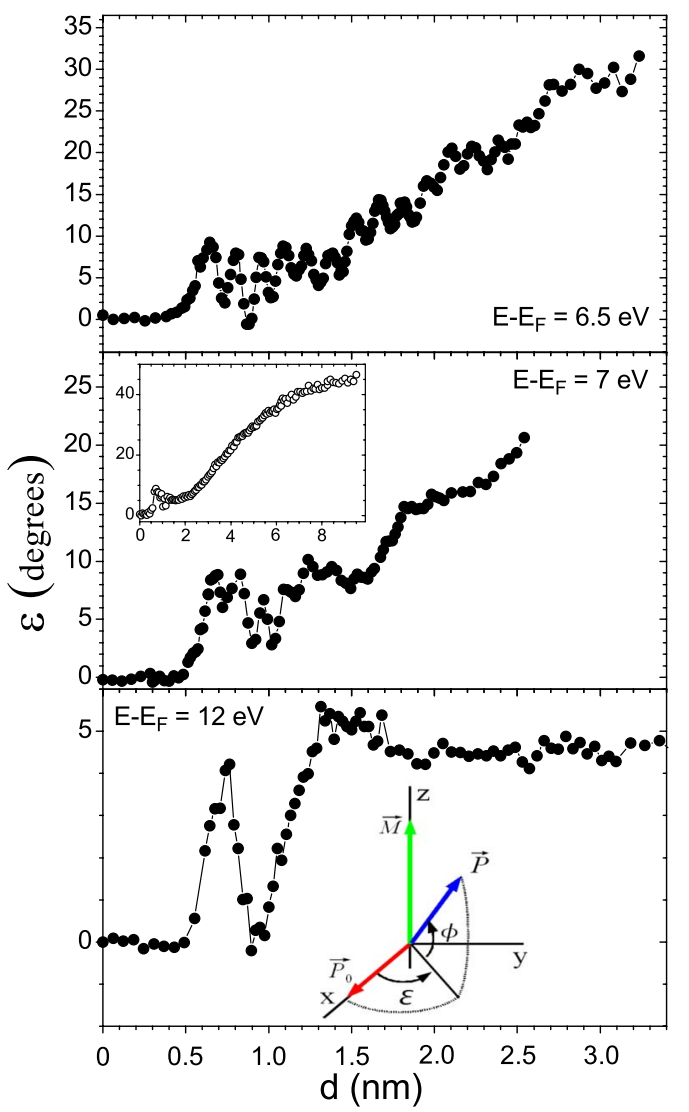

FIG. 2. (Color online) The precession angle $\varepsilon$ as a function of the Fe film thickness for three different primary electron energies $E-E_{F}$. The inset in the middle panel shows $\varepsilon$ for a larger Fe thickness range. The lines are guides to the eye. The inset in the bottom panel shows the two types of motions of the spin-polarization vector: a precession about the magnetization $\mathbf{M}$ by an angle $\varepsilon$ and a rotation by an angle $\phi$ in the plane spanned by $\mathbf{P}$ and $\mathbf{M}$.

to see two oscillation periods: a longer one with wavelength $2 \pi /\left|k^{\uparrow}-k^{\downarrow}\right|$ and a shorter one with wavelength $2 \pi /\left(k^{\uparrow}+k^{\downarrow}\right)$ with $k^{\uparrow, \downarrow}$ the spin-dependent wave vector. However, the longer period is usually too long (of the order of $100 \mathrm{ML}$ ) to be seen in the measurements, so that in practice only the short oscillation period is visible. This period is shown as a function of the primary electron energy in the inset of the bottom panel in Fig. 3.

What are the possible origins of the ML oscillations? One might think that the variations of the surface morphology (with a period of one ML) should lead directly to the ML oscillations via the interference of electrons being reflected from film terraces differing by $1 \mathrm{ML}$ in thickness. For this to happen, however, the electrons have to fulfill the anti-Bragg condition, which is not the case in the energy range where the ML oscillations are visible. Variations of the saturation magnetization such as, for instance, those proposed for the system oxygen on $\mathrm{Fe}(001)$ (Ref. 16) or variations of the remanent magnetization are excluded as well. They should be independent of the electron energy and should therefore occur at any electron energy.

As a possible origin of the ML oscillations, we suggest an oscillation of the surface-lattice parameter during Fe deposi-

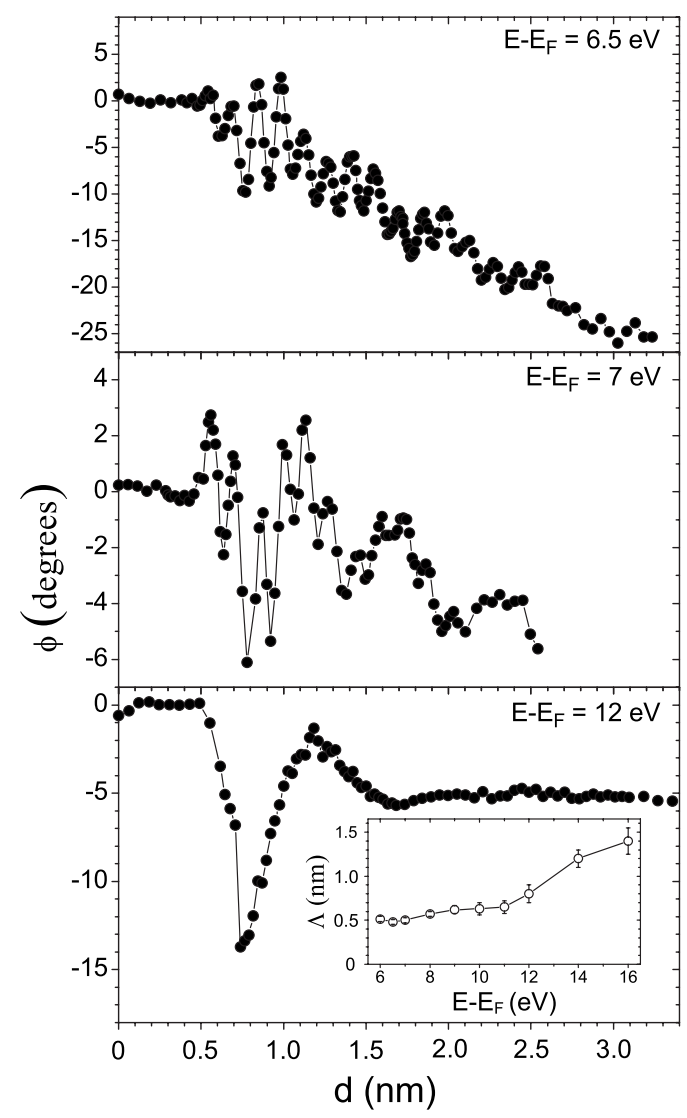

FIG. 3. The rotation angle $\phi$ as a function of the Fe film thickness for three different primary electron energies $E-E_{F}$. The inset in the bottom panel shows the period $\Lambda$ of the long-period oscillations as a function of the primary electron energy. The lines are guides to the eye.

tion. In fact, thin films grown on a substrate are generally subject to strain arising from different bulk lattice parameters of the film and the substrate. Two relaxation mechanisms can relieve the strain. Up to a critical thickness, the film grows pseudomorphically on top of the substrate, so that relaxation can only occur at the incomplete surface layer. The surface lattice can relieve the strain by a relaxation of the atomic positions at island edges. ${ }^{17}$ Consequently, the strain relaxation is strongest for half-filled layers with a maximum of the number of islands. Therefore, with increasing film thickness the average lattice parameter varies in an oscillatory fashion with ML periodicity. For thicknesses above the critical value, the strain is relieved by the creation of interfacial dislocations in the film. ${ }^{18}$ Thus, the ML oscillations are superimposed on a constant background strain up to the critical thickness and on a monotonically increasing relaxation of the lattice parameter above it. Our data suggest such an interpretation. Considering, for instance, the data at $6.5 \mathrm{eV}$, we see that the ML oscillations have a constant background value up to a thickness of about $1.3 \mathrm{~nm}$, while the latter increases for higher coverages.

Unfortunately, no structural data are available in the literature for $\mathrm{Fe}$ films on $\mathrm{Ag}(001)$ proving the existence of surface-lattice parameter oscillations. Moreover, one might argue that the misfit of the bulk lattice parameters of $0.4 \%$ is 
too small to induce a significant oscillation amplitude. However, even for homoepitaxial systems the surface strain for islands is considerably different from flat surfaces, ${ }^{19}$ in particular for small islands, and thus leads to oscillations of the surface-lattice parameter during growth. In addition, even a slight contamination of the film surface during growth, which we actually detect, can induce remarkable oscillations of the surface-lattice parameter. ${ }^{20}$

We emphasize that the amplitude of the ML oscillations depends strongly on the Ag crystal used in the experiments. Whereas the long-period oscillations as well as the overall change of the spin-motion angles appear always with the same amplitude, whatever the Ag crystal, no ML oscillations could be observed on some Ag crystals. This dependence of the ML oscillations on the substrate points also to the variation of the surface-lattice parameter as an origin of the ML oscillations. In fact, the amplitude of the surface-lattice parameter oscillations depends on the nucleation density of the film, ${ }^{21}$ a quantity that may vary strongly from one Ag substrate to another as it depends in particular on the terrace sizes of the substrate.

How can we understand in the above context the fact that the ML oscillations as well as the overall change above the critical thickness appear only in a limited electron energy range? It is plausible that variations of the lattice parameter can lead to a change of the electron reflection amplitude. From scattering theory it is known that the total scattering amplitude can be deduced from the partial scattering amplitudes for the different angular momenta. ${ }^{22}$ The crucial point is that even if the change of the lattice parameter results only in small variations of the partial scattering amplitudes, the total scattering amplitude and thus $\varepsilon$ and $\phi$ of the reflected electrons may vary considerably under certain circumstances. Such a circumstance occurs when the partial scattering phases are such that the modulus of the total scattering amplitude becomes small (the so-called generalized Ramsauer-Townsend effect ${ }^{23}$ ). In fact, the reflected intensity in our experiments exhibits a pronounced minimum in the energy range of interest. Moreover, calculations made in the atomic limit do indeed predict a Ramsauer-Townsend effect in the same energy range. ${ }^{24}$ In order to further corroborate this interpretation, spin-dependent LEED calculations have to be performed in the future.

In conclusion, beside oscillations of the electron-spin motion due to quantum interference, oscillations of ML periodicity have also been observed in Fe films on $\operatorname{Ag}(001)$ in spin-polarized electron reflection experiments. We attribute the latter to periodic variations of the surface-lattice parameter of Fe during growth. Consequently, our experiment directly evidences an extreme sensitivity of the spin-transfer effect, i.e., the transfer of spin-angular momentum from the incident spin-polarized electrons to the magnetization, on the morphology and structure of the ferromagnetic film at certain electron energies.

We would like to thank D. Sébilleau for communicating his results to us.
${ }^{1}$ M. N. Baibich, J. M. Broto, A. Fert, F. Nguyen Van Dau, F. Petroff, P. Eitenne, G. Creuzet, A. Friederich, and J. Chazelas, Phys. Rev. Lett. 61, 2472 (1988).

${ }^{2}$ G. Binasch, P. Grünberg, F. Saurenbach, and W. Zinn, Phys. Rev. B 39, 4828 (1989).

${ }^{3}$ J. C. Slonczewski, J. Magn. Magn. Mater. 159, L1 (1996).

${ }^{4}$ L. Berger, Phys. Rev. B 54, 9353 (1996).

${ }^{5}$ E. B. Myers, D. C. Ralph, J. A. Katine, R. N. Louie, and R. A. Buhrman, Science 285, 867 (1999).

${ }^{6}$ W. Weber, S. Riesen, and H. C. Siegmann, Science 291, 1015 (2001).

${ }^{7}$ L. Joly, J. K. Ha, M. Alouani, J. Kortus, and W. Weber, Phys. Rev. Lett. 96, 137206 (2006).

${ }^{8}$ L. Joly, L. Tati-Bismaths, and W. Weber, Phys. Rev. Lett. 97, 187404 (2006).

${ }^{9}$ L. Joly, L. Tati Bismaths, F. Scheurer, and W. Weber, Phys. Rev. B 76, 104415 (2007).

${ }^{10}$ S. Tanuma, C. J. Powell, and D. R. Penn, Surf. Interface Anal. 17, 927 (1991)

${ }^{11}$ G. C. Smith, H. A. Padmore, and C. Norris, Surf. Sci. 119, L287 (1982).

${ }^{12}$ B. Heinrich, S. T. Purcell, J. R. Dutcher, K. B. Urquhart, J. F.
Cochran, and A. S. Arrott, Phys. Rev. B 38, 12879 (1988).

${ }^{13}$ P. J. Schurer, Z. Celinski, and B. Heinrich, Phys. Rev. B 51, 2506 (1995).

${ }^{14}$ H. Li, Y. S. Li, J. Quinn, D. Tian, J. Sokolov, F. Jona, and P. M. Marcus, Phys. Rev. B 42, 9195 (1990).

${ }^{15}$ M. Stampanoni, A. Vaterlaus, M. Aeschlimann, and F. Meier, Phys. Rev. Lett. 59, 2483 (1987).

${ }^{16}$ M. Nyvlt, F. Bisio, J. Franta, C. L. Gao, H. Petek, and J. Kirschner, Phys. Rev. Lett. 95, 127201 (2005).

${ }^{17}$ J. Fassbender, U. May, B. Schirmer, R. M. Jungblut, B. Hillebrands, and G. Güntherodt, Phys. Rev. Lett. 75, 4476 (1995).

${ }^{18}$ A. Chambers and D. C. Jackson, Philos. Mag. 31, 1357 (1975).

${ }^{19}$ O. V. Lysenko, V. S. Stepanyuk, W. Hergert, and J. Kirschner, Phys. Rev. Lett. 89, 126102 (2002).

${ }^{20}$ P. Turban, L. Hennet, and S. Andrieu, Surf. Sci. 446, 241 (2000).

${ }^{21}$ P. Müller, P. Turban, L. Lapena, and S. Andrieu, Surf. Sci. 488, $52(2001)$.

${ }^{22}$ J. B. Pendry, Low Energy Electron Diffraction (Academic, London, 1974)

${ }^{23}$ J. J. Barton, Z. Hussain, and D. A. Shirley, Phys. Rev. B 35, 933 (1987).

${ }^{24}$ D. Sébilleau (private communication). 